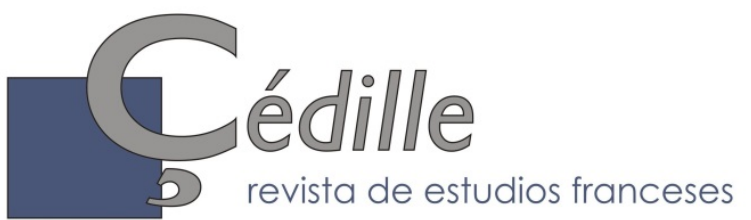

ISSN: $1699-4949$

no 17 (otońo de 2020)

Monografías 10

Les euphémismes dans les médias : entre voilements, démasquages et discours qui les traversent Montserrat López Díaz \& Annabelle Seoane, éditrices scientifiques

\title{
Euphémisation, conflit nominatif et ethos dans les entretiens politiques médiatiques
}

\author{
Ruggero DRUETTA \\ Università degli Studi di Torino \\ ruggero.druetta@unito.it \\ ORCID: 0000-0002-6117-3437
}

\section{Resumen}

Este artículo analiza la eufemización que se da en las entrevistas políticas mediáticas como resultado de un conflicto nominativo. Llamamos conflicto nominativo a una secuencia interactiva estructurada en la cual un locutor cuestiona y sustituye un término utilizado por otro, hasta llegar a un nombre consensuado. Resulta así un número considerable de nombres alternativos, vinculados a puntos de vista opuestos, lo que permite observar el dialogismo inherente al conflicto nominativo y a la producción de eufemismos destinados a establecer o restablecer la conformidad del discurso con una norma discursiva que refleja los valores de una comunidad. Esto trae un beneficio para el ethos del locutor, que se ve valorado. Los fenómenos suprasegmentales y coverbales constituyen marcas auxiliares de este trabajo dialógico e indicios auxiliares de relevancia.

Palabras clave: Eufemismo. Retórica. Ethos. Multimodalidad. Discurso político.

\section{Résumé}

Cet article analyse l'euphémisation apparaissant dans les interviews politiques médiatisées comme aboutissement d'un conflit nominatif. Nous appelons conflit nominatif une séquence interactive structurée dans laquelle un locuteur conteste et remplace un terme utilisé par un autre, jusqu'à parvenir à une nomination consensuelle. Il en ressort un nombre important de nominations alternatives, rattachées à des points de vue concurrents, qui permet d'observer le dialogisme inhérent au conflit nominatif et à la production d'euphémismes visant à établir ou rétablir une conformité du discours à une norme discursive reflétant les valeurs d'une communauté. Cela apporte un bénéfice éthotique au locuteur, qui s'en trouve valorisé. Les phénomènes suprasegmentaux et coverbaux constituent des marques auxiliaires de ce travail dialogique et des indices auxiliaires de saillance.

Mots clé : Euphémisme. Rhétorique. Ethos. Multimodalité. Discours politique.

* Artículo recibido el 17/10/2019, aceptado el 11/04/2019. 


\begin{abstract}
This paper analyses euphemisms found in political interviews in the media as an issue of a nominative conflict. Nominative conflict is an interactive structured sequence where a speaker questions a term proposed by a conversation partner and where she proposes another term in turn, until s/he finds a consensual nomination. This structure intrinsically brings out many alternative terms, which depend on competing points of view: this allows us to notice the dialogism inherent in nominative conflict as well as in the production of euphemisms. This production aims to establish or re-establish the discourse compliance with a discursive norm reflecting the values of a community. That provides an ethotic benefit to the speaker, whose image is enhanced. Suprasegmental and coverbal phenomena represent auxiliary evidence of the dialogic work and auxiliary marks of saliency.
\end{abstract}

Keywords: Euphemism. Rhetoric. Ethos. Multimodality. Political discourse.

\title{
0. Introduction
}

Au début des entretiens politiques, ou après chaque nouvelle question de la part du journaliste, on peut souvent assister à une négociation interlocuteurs (dialogisme externe) au sujet de la nomination ${ }^{1}$ appropriée du référent discursif, le journaliste proposant généralement des nominations axiologiquement et/ou connotativement plus marquées (manifestant une orientation dysphémique et, en tout cas "politiquement incorrectes »), alors que le politique reformule, lui, de manière plus euphémique et "politiquement correcte", cette nomination renvoyant à une source énonciative collective (celle du gouvernement, des décideurs, du parti politique...) à laquelle il revendique son appartenance. Ces séquences de rectification manifestent donc un dialogisme interdiscursif (par rapport aux discours antérieurs de la même formation politique ou d'autres sources énonciatives) et intralocutif (les prises de parole antérieures de l'homme politique), qui se greffent sur le dialogisme interlocutif (dialogisme externe) que nous venons d'évoquer.

Dans cet article ${ }^{2}$, nous essaierons d'analyser la négociation nominative en tant que lieu discursif où s'affrontent polyphoniquement plusieurs points de vue antagonistes : après avoir introduit les notions de nomination et de conflit nominatif et avoir montré le rapport entre nomination, euphémisme et polyphonie, via la notion de point de vue, nous présenterons notre approche méthodologique, notamment en ce qui concerne la multimodalité et la représentation des euphémismes. Nous montrerons l'intérêt d'appréhender le fonctionnement de ces notions dans le domaine politique, à travers l'analyse de quelques cas de conflit nominatif dans des interviews politiques.

\footnotetext{
${ }^{1}$ Nous expliquons infra, $\$ 1$. notre choix du terme nomination de préférence à dénomination.

${ }^{2}$ Travail réalisé dans le cadre des projets de recherche FFI2013-42249P et FFI2017-85141P (FEDER, Ministerio de Ciencia, Innovación y Universidades, AEI).
} 


\section{Nomination euphémique et polyphonie}

\subsection{Nomination, catégorisation et référenciation}

Puisque l'acte d'euphémisation correspond au remplacement d'un lexème par un autre, jugé moins choquant, l'approche de l'euphémisme exige que nous nous penchions également sur l'opération de référenciation, à savoir sur la possibilité, pour un mot, de désigner le référent d'un autre à travers l'imposition d'une relation de synonymie déjà existante (catachrèses) ou attribuée discursivement. Afin d'en rendre compte correctement, nous allons d'abord passer en revue les notions de dénomination et désignation (Kleiber, 1984), dont la distinction s'avère essentielle pour discriminer deux modalités d'accès au référent, et nous allons plaider ensuite en faveur du terme hyperonyme de nomination (Siblot, 2001) comme mieux adapté à la situation discursive qui est la nôtre.

La dénomination pose un lien référentiel stable et une relation métalinguistique au référent, par l'intermédiaire du concept (le signifié). Sa stabilité vient d'une opération de nomination préalable, ce qui entraîne la nécessité d'un apprentissage avant de pouvoir l'utiliser de manière consensuelle, ainsi que de son stockage dans la compétence lexicale du locuteur: en effet la référenciation est effectuée par voie lexématique.

La désignation, en revanche, pose un lien occasionnel, en discours, qui ne repose ni sur une nomination ni sur un apprentissage préalable. S'agissant d'un phénomène non pas de compétence, mais de performance, sa modalité propre de réalisation et de référenciation est syntagmatique. Ce sont les « descriptions définies " de la tradition philosophique ( $c f$. notamment Frege, 1892 ; Russell, 1905; Strawson, 1950 ; Kripke, 1972), les "expressions complexes » de Kleiber (1984: 81) ou encore les "périphrases" de la rhétorique, et il est tout à fait possible que la désignation constitue cet acte premier de "baptême " suite auquel le statut de dénomination pourra se mettre en place, au fur et à mesure que l'utilisation de telle expression se stabilise et se répand auprès du plus grand nombre.

La convertibilité de la désignation en dénomination, d'une part, et la possibilité concrète, pour le locuteur, d'utiliser indifféremment l'une ou l'autre des deux modalités dans sa prise de parole, d'autre part, nous suggèrent d'utiliser un terme hyperonyme des deux et qui permette, de plus, de souligner le positionnement $\mathrm{du}$ locuteur vis-à-vis des référents dénommés ou désignés, ainsi que la prise en charge des contenus énonciatifs rattachés à ses choix langagiers ${ }^{3}$. Nous adhérons à la proposition de Siblot (2001), qui suggère de distinguer entre le plan de la langue, qui enregistre des associations stables entre unités lexicales et sens, pour lesquelles on utilisera le terme de dénomination, et le plan du discours, où l'utilisation d'une expression lexicale n'est pas forcément conventionnelle, calée sur des usages antécédents, mais cor-

${ }^{3}$ C'est la notion de point de vue (désormais : PDV) telle que définie par Ducrot (1984) et Rabatel (2003, 2005). 
respond davantage au rapport actuel du locuteur à la réalité. Dans ce cas, on parlera de nomination, terme par lequel on focalise l'aspect individuel de l'opération énonciative de référenciation et l'engagement personnel du locuteur, à travers le point de vue que celui-ci exprime dans et par ses choix langagiers. Dans la suite de ce travail, nous allons donc adopter le terme de nomination pour nous référer aux unités lexicales en compétition dans les échanges conflictuels des interviews politiques. Ce terme indique pour nous la dimension processuelle de l'emploi discursif d'une expression simple (mot) ou complexe ( $\mathrm{SN}$ ou périphrase) à finalité dénominative ou désignative qui comporte des opérations de réglage du sens lors de sa mise en discours ${ }^{4}$. Ce choix entraîne deux conséquences : d'une part, le degré de complexité des expressions nominatives détermine un accès référentiel plus ou moins aisé en fonction du consensus autour de cette catégorisation. D'autre part, la nomination - et la catégorisation référentielle qui en découle - est la manifestation la plus directe du PDV du locuteur ou de l'issue de la confrontation entre PDV aboutissant in fine à telle nomination, dans la mesure où le nom ou le syntagme produit se situent par rapport au paradigme ouvert des expressions pouvant commuter avec ceux-ci.

\subsection{Conflit de points de vue et polyphonie}

Dans l'interaction orale, la composition des PDV des interlocuteurs in praesentia et d'énonciateurs divers et variés in absentia suscite une polyphonie abondante qui se manifeste sur le plan syntagmatique notamment par des séquences de recherche lexicale visant à sélectionner la nomination la mieux adaptée non seulement par rapport au référent discursif, mais aussi et surtout par rapport aux PDV en jeu, étant donné que, ici comme ailleurs, "on euphémise sous la pression d'autrui et à l'intention d'autrui." (Bonhomme, 2005 : 240). Lorsqu'elle est linéarisée sur l'axe syntagmatique, cette recherche donne lieu à des entassements nominatifs, des "piles " paradigmatiques (Gerdes \& Kahane, 2009), éventuellement accompagnées de disfluences verbales, de commentaires métalinguistiques et de marqueurs de reformulation, tout cela permettant d'observer le travail de formulation sur le vif. La fonction de ces séquences est d'ajuster la nomination à la catégorisation du référent visé, dans la mesure où celle-ci correspond à une norme sociale à laquelle le locuteur veut adhérer ou par rapport à laquelle, en tout cas, il doit se positionner. Que ce positionnement prenne la forme du conflit nominatif ( $c f$. infra, $\$ 2.1$.) ou pas, le choix d'une nomination parmi d'autres revêt une importance argumentative primordiale, qui peut éventuellement se doubler d'une argumentation explicite justifiant l'expression retenue.

\footnotetext{
${ }^{4}$ Nous précisons que nous appliquons le terme nomination aussi bien aux noms qu'aux adjectifs, verbes et adverbes.
} 


\subsection{L'euphémisme comme issue possible du conflit}

La polyphonie résultant de la multiplicité de PDV actualisés dans des discours divergents et des nominations différentes peut déboucher, dans l'interaction orale, sur l'affrontement agonal ou, tout en ayant conscience de ce "many perspectives view" (Clark, 1997), sur la recherche d'une nomination partagée, en mesure de dépasser et d'apaiser la tension attachée aux nominations alternatives. C'est ici que l'euphémisme peut apparaître en tant que nomination détensive par rapport à une nomination malséante et tendue (Bonhomme, 2005), voire carrément taboue, tout comme le politiquement correct (López Díaz, 2014), qui participe en effet de cette orientation. Le fait que l'euphémisme puisse faire l'objet d'un conflit nominatif ( $c f$. infra, $\$$ 2.1.) atténue, sans l'infirmer pour autant, l'observation de Jaubert (2008), pour qui l'euphémisme repose sur un dialogisme interdiscursif, tandis que la litote repose sur un dialogisme interlocutif. En effet, la valeur lénifiante de l'euphémisme se fonde bien sur le dialogisme interdiscursif, puisqu'elle se manifeste dans le contraste par rapport à ce qui aurait pu être dit dans un discours reflétant un autre PDV, tandis que le conflit nominatif avec l'interlocuteur in praesentia vise à défendre la légitimité d'une telle nomination. Il apparaît cependant que, dans le cas de l'interaction orale, il peut bien y avoir dialogisme interlocutif externe autour de l'euphémisme.

Quant au mécanisme de contournement du conflit potentiel autour de la nomination d'un référent tendu, le locuteur euphémisant choisit une nomination indirecte, soit en modifiant la catégorisation sémantique habituelle et, partant, la référenciation, soit en opacifiant carrément la catégorisation, ce qui rend l'accès au référent moins immédiat. Dans les deux cas, l'abandon du signifiant habituel et le brouillage du parcours référentiel aboutissent - en même temps et dans une plus ou moins grande mesure - d'une part à la réduction ou à la neutralisation de la tension et, d'autre part, à la mélioration axiologique, qui sont les deux dimensions fondamentales de tout euphémisme (Bonhomme, 2005).

\section{Dans l'interview politique}

L'entretien politique est un lieu d'observation privilégié de l'émergence des figures rhétoriques, d'une part parce que le débat public constitue l'un des champs d'application fondamentaux de l'art rhétorique et, d'autre part, parce que cela nous donne la possibilité d'observer ces figures à l'oral, avec toutes les manifestations qui accompagnent leur performance, d'un point de vue verbal (piles paradigmatiques, amorces de mots, hésitations, marqueurs de reformulation,...), suprasegmental (saillances intonatives et micro-variations prosodiques) et coverbal (gestes volontaires ou involontaires accompagnant l'élocution, dimension de l'Actio oratoire), ce qui devrait aboutir à une meilleure appréhension de ces phénomènes.

Concernant l'euphémisme, les interviews et entretiens politiques sont des objets idéals pour cette observation, car ils sont intrinsèquement "euphémismogènes " (Druetta, 2009) et, d'un point de vue énonciatif, l'étude de ces productions orales 
permet aussi d'observer les différentes formes de dialogisme : en effet, le positionnement idéologique et discursif des hommes politiques est affiché et notoire, et ils n'ont de cesse de le manifester publiquement à chacune de leurs prises de parole, en précisant les points de divergence avec d'autres positionnements. Par ailleurs, la présence d'un collectif derrière le politique (le parti, les militants) se traduit dans des pratiques matérielles, discursives et langagières partagées et, parmi celles-ci, il faut mentionner tout particulièrement la présence de textes et de personnalités de référence, servant de modèles, ainsi que la pratique de rédiger des argumentaires destinés justement à la prise de parole publique, qui offrent des nominations toutes faites pour les référents discursifs de l'actualité dont les politiques sont amenés à débattre. Enfin, la dimension agonale de l'engagement politique et du genre de l'entretien politique est le cadre idéal pour le surgissement de nominations problématiques qu'il s'agit de mitiger ou de désamorcer à des fins argumentatives (conflit nominatif, $\$ 2.1$.) ou éthotiques $(\$$ 2.2.).

\subsection{Le format du conflit nominatif}

D'un point de vue discursif, le conflit nominatif consiste en une séquence dialogale de négociation de la nomination ou de la qualification d'un référent discursif, pouvant être initiée par l'un des interlocuteurs et suscitant une boucle de rétroaction (feedback) négative de la part de l'autre locuteur, qui propose une nomination ou une qualification alternative. La négociation peut être abandonnée ou poursuivie jusqu'à l'obtention d'un consensus ou à l'abandon du sujet ("victoire " de l'un des deux ou diversion). La polyphonie se manifeste dans les nominations alternatives qui se succèdent in praesentia (issues d'un seul locuteur ou des deux en confrontation), ou dans la recherche lexicale in absentia, (éventuellement manifestée par des disfluences verbales et coverbales).

Appliquée au cas de l'euphémisme, la dynamique prototypique du conflit nominatif est généralement initiée par le journaliste, qui utilise une nomination et/ou une qualification dysphémique ou problématique pour l'une des faces (positive ou négative) du politique. Celui-ci réagit en contestant le journaliste et en proposant une nomination euphémique visant à réparer cette atteinte; le conflit continue tant que l'un des deux n'a pas réussi à imposer sa propre nomination et, à travers celle-ci, son PDV. L'exemple suivant ${ }^{5}$, que nous avons considérablement écourté pour des raisons de place, illustre ce conflit, au bout duquel le qualificatif d'insincère est remplacé par la désignation prédicative de bonne guerre. La durée totale de la séquence est de 4 min. 30, sur une durée totale de l'entretien de 20 minutes, ce qui signifie que le con-

\footnotetext{
${ }^{5}$ Interview de Manuel Valls par Jean-Jacques Bourdin, le 4 juillet 2017, BFM et RMC. Nous utilisons une transcription orthographique, sans ponctuation et sans majuscules. En ce qui concerne les signes additionnels, le « + " indique une pause, le tiret «- " les amorces de mot, les «: " l'allongement syllabique. Le soulignement indique le chevauchement de parole entre deux locuteurs. Les initiales conventionnelles J (journaliste) et $\mathrm{P}$ (politique) désignent les locuteurs.
} 
flit nominatif a occupé $20 \%$ de l'entretien. On remarque aussi que, pendant le conflit, le recours à des expressions métalinguistiques est fréquent (je réfute totalement ce terme d'insincérité; il faut pas dire n'importe quoi; je n'accepte pas moi le terme d'insincérité) : le locuteur doit en effet argumenter en faveur du changement nominatif.

\section{[1]}

$\mathrm{J}$ il manque au moins + huit milliards d'euros dans les caisses de l'état ++ c'est ce que dit + la Cour des Comptes budget insincère ce budget c'est vous qui l'avez préparé ++

$P \quad$ je je je refuse je je refuse $[\ldots]$ je réfute totalement ce terme d'insincérité + qu'i- qu'il y ait des difficultés pour boucler le budget c'est incontestable [...] il y a un certain nombre de difficultés mais il faut pas dire n'importe quoi $+[\ldots]$ je n'accepte pas moi le terme d'insincérité [...] c'est de bonne guerre $[\ldots]$ mon expérience m'amène à penser que c'est bien joué (BFM et RMC, 04/07/2017).

\subsection{Enjeux éthotiques}

Il n'est pas nécessaire de rappeler ici qu'à côté de l'argumentation rationnelle, les dimensions du pathos et de l'éthos contribuent à asseoir la crédibilité de l'orateur et entraînent l'adhésion à ses propos. La preuve éthique s'avère particulièrement importante dans le cas des politiques, car le caractère public de leur engagement, ainsi que leurs prises de parole et leurs passages à l'antenne fréquents, contribuent à créer un ethos préalable stable, qui interagit avec l'ethos discursif et surdétermine la réception de leurs discours. Cet "hologramme expérientiel» (Auchlin, 2000) doit être continuellement entretenu, au risque de se dissoudre, et l'euphémisme y contribue de manière essentielle, dans la mesure où la capacité de rester calme face aux adversités, de garder un vocabulaire et un discours de sagesse et de modération dans toutes les occasions étaie tout particulièrement la dimension éthique de la phronésis, considérée comme essentielle chez un représentant politique ayant des fonctions de gouvernement ou pouvant y prétendre.

L'adoption du politiquement correct ou de tout autre " code » ou " registre " (ou "répertoire») langagier dans une situation publique implique par ailleurs une polyphonie et un positionnement par rapport à la multiplicité des sources énonciatives et des formations discursives agissant dans l'espace public, ainsi que des ethé qui leur sont associés, par-delà même les attitudes concrètes des locuteurs in praesentia. À cause du poids du conformisme social qui lui est attaché, le simple recours à l'euphémisme et au politiquement correct (tels que codifiés et partagés, à un moment donné, par une communauté de locuteurs) vaut donc ethos : le locuteur peut très bien avoir un ethos performé assez agressif, mais incarner, par son comportement verbal, l'ethos de modération du politiquement correct, qu'il pourra, à l'occasion, revendiquer pour lui. 


\section{Corpus et méthodologie}

Pour notre analyse, nous utilisons des interviews diffusées à la télévision ou à la radio, et dont la vidéo est disponible au téléchargement, de manière à pouvoir disposer d'un enregistrement de bonne qualité. Étant donné notre volonté d'analyser la composante coverbale, nous privilégions les interviews comportant peu de changements de caméras. De ce point de vue, les plans fixes des interviews radio s'avèrent être les meilleurs, car il n'y a jamais d'interruption dans l'enregistrement des gestes. Nous n'avons pas constitué de corpus au sens strict, mais nous avons recueilli une quarantaine d'interviews de mars à juillet 2017 et nous en avons extrait des séquences significatives de conflit nominatif. Pour des raisons de place, nous n'en présentons que deux.

\subsection{Les traces syntagmatiques du dialogisme}

La confrontation des PDV sous-jacents aux différentes nominations dans le discours du locuteur euphémisant laisse des traces observables sur l'axe syntagmatique. Celles-ci sont de deux types. Le premier type fait appel à une caractéristique de base de toute production orale spontanée, à savoir les disfluences (pauses vides et remplies, amorces de mots, répétitions, listes paradigmatiques sur une place syntaxique), qui sont la conséquence - généralement involontaire - des opérations cognitives liées à la programmation en temps réel du discours (Druetta, 2009, 2015). Normalement inaperçues, du fait de leur caractère constitutif, tel un bruit de fond, celles-ci deviennent significatives et se font remarquer dans la mesure où il y a des variations importantes dans leur fréquence, ce qui détermine une accumulation, un surplus de matériel verbal (entassement paradigmatique, Druetta, 2009). Si l'on admet l'hypothèse que la production figurale mobilise des ressources cognitives importantes, liées non seulement à la recherche lexicale, mais aussi au calcul de l'effet sur l'interlocuteur, on comprend que les disfluences qui s'en ensuivent peuvent avoir une valeur diagnostique importante. En effet, la disfluence apparaît lorsque le locuteur essaie de remplacer une nomination dysphémique que celui-ci récuse mais qui ne s'en impose pas moins à lui (le reparandum, dans la terminologie de Shriberg, 1994) et se termine lorsqu'il aboutit à une nomination euphémique satisfaisante (le repair de Shriberg). À ce titre, on remarque aussi que, chez certains locuteurs, les disfluences peuvent entrer dans une stratégie de saillance. Elles sont alors produites délibérément, afin d'attirer l'attention du destinataire sur la production de la figure, à des fins argumentatives ou éthotiques.

Le deuxième type de traces concerne des marqueurs métalinguistiques autonymiques de reformulation ou de balisage (marqueurs de reformulation, Druetta, 2009), qui visent spécifiquement à délimiter le surgissement de la figure et à focaliser l'attention du destinataire sur celle-ci ainsi qu'à signaler le travail accompli sur la nomination. Il s'agit d'expressions telles que : j'allais dire, c'est un euphémisme, pardon 
pour les oreilles chastes, etc., ainsi que de phénomènes prosodiques et gestuels connexes.

C'est pourquoi, dans notre analyse, nous allons signaler et regrouper systématiquement les éléments pouvant être rattachés aux entassements paradigmatiques et aux marqueurs de reformulation, afin de déterminer les saillances segmentales et suprasegmentales de la figuralité, ainsi que la concordance ou discordance éventuelles entre celles-ci.

\subsection{Analyse multimodale}

Nous considérons la production langagière comme une activité complexe, s'appuyant à la fois sur des aspects neurologiques et cognitifs, d'une part, physiologiques et corporels, d'autre part : l'activité cérébrale consciente, bien sûr, mais aussi les organes phonatoires, le schéma corporel et la gesticulation, la proximité avec l'interlocuteur, le regard, etc. Nous pouvons regrouper ces différentes composantes en trois strates interconnectées mais répondant chacune à des contraintes et à des logiques propres (principe de "Souveraineté-Association », cf. Martin, 2011) : la strate verbale segmentale, la strate prosodique suprasegmentale et la strate mimogestuelle coverbale.

Afin d'annoter les différentes composantes multimodales, nous nous sommes servi du logiciel d'analyse prosodique PRAAT $^{6}$, ainsi que du script Prosogram ${ }^{7}$, dans lesquels nous avons aligné la transcription des séquences analysées au tracé prosodique de la fréquence fondamentale et de l'intensité (ou du spectrogramme, en fonction des cas). Nous avons ajouté dans cette transcription une annotation manuelle de la gestualité coverbale, en utilisant autant de "tires" (lignes de transcription dans PRAAT) que nous avons distingué de segments corporels, alignées elles aussi à la parole transcrite, ce qui nous a permis de relever la cooccurrence ou le déphasage de plusieurs marques de saillance. Nous avons ainsi identifié cinq segments corporels : tête, yeux, bouche, mains, buste.

Concernant l'annotation de la gestualité, il nous semble bon de préciser que notre approche de la gestualité coverbale se veut résolument distincte de la « synergologie " (p. ex. Turchet, 2009), car nous ne nous aventurons pas dans une lecture à visée psychologique des mouvements du locuteur se déployant de façon totalement autonome et inconsciente par rapport au contenu de son vouloir-dire. Nous considérons la gestualité produite simultanément à la parole et, en particulier, dans la proximité immédiate du surgissement des figures, comme l'ombre portée par le discours sur le cinétisme de l'orateur, que ce soit de façon volontaire ou involontaire. Nous nous y intéressons donc dans la mesure où celle-ci participe de l'action de communication, comme le font, p. ex. des chercheurs comme Bouvet \& Morel (2002) ou

\footnotetext{
${ }^{6}$ http://www.fon.hum.uva.nl/praat.

${ }^{7}$ https://sites.google.com/site/prosogram/home.
} 
Guaïtella (1995). C'est la spécificité de la performance orale, qui fait traditionnellement l'objet de l'Actio oratoire, la présence physique de l'orateur venant appuyer ou contredire son discours verbal, à travers la coordination ou l'incohérence de ses mouvements de ponctuation rythmique, de figuration gestuelle, de son occupation de l'espace, de son assurance corporelle par rapport à ce qu'il énonce discursivement.

\subsection{Position relative des euphémismes et représentation par tenseur binaire radical}

À l'opposé des approches substitutives du fait rhétorique, fondées d'une manière ou d'une autre sur le postulat de l'orthonymie, de l'existence d'un degré zéro de la nomination, et sur celui de l'immanence du sens, la perspective énonciative dans laquelle nous nous inscrivons fait fi de ces postulats et installe le dialogisme au cœur de la signification. Celle-ci dépend de la relation du sujet parlant avec le monde et ses praxis (Détrie, 2001), ainsi qu'avec les autres discours et leurs locuteurs/énonciateurs, et associe de ce fait toute nomination concrète à un PDV particulier pouvant faire l'objet d'une négociation sociale - consensuelle ou agonale - pour parvenir à un "sens " partagé. Ainsi, pour un référent donné, y aura-t-il plusieurs possibilités de nominations - euphémiques et dysphémiques - et leur valeur individuelle n'apparaîtra que par l'établissement d'une relation entre les nominations concurrentes. Par conséquent, pour représenter les rapports dynamiques entre les différentes nominations qui composent un conflit nominatif dans nos exemples, nous avons réalisé un schéma constitué d'une ligne horizontale sur laquelle nous avons posé deux vecteurs orientés, illustrant les deux cinétismes successifs opérant dans l'euphémisme : la détension et la mélioration ( $\$$ 1.3. ; cf. Bonhomme, 2005). Suivant l'orientation conventionnelle de gauche à droite, le premier cinétisme est dé-tensif. Le premier ressort de l'euphémisation consiste en effet à réduire, de manière plus ou moins marquée, la tension dysphémique. Le point d'aboutissement de ce cinétisme décroissant n'épuise cependant pas la dynamique euphémique, qui, à partir de là, est relayée par un cinétisme mélioratif croissant, qui consiste à attribuer des connotations positives au référent à travers un lexique axiologiquement mélioratif. Sur la ligne horizontale, nous avons ensuite placé les différents procédés euphémiques, en fonction de leur position respective par rapport aux deux cinétismes de base. Lors de l'analyse, nous avons rangé les nominations concurrentes mises en jeu par l'interaction verbale en correspondance des différents points de cet espace tridimensionnel. Il en ressort une cartographie permettant de mesurer les dynamiques entre les prises de parole successives et le travail accompli sur les nominations par les locuteurs impliqués. La représentation schématique que nous proposons se rapproche du tenseur binaire radical de Guillaume (1929, 1973), qui constitue en effet un mécanisme fondamental du langage. 


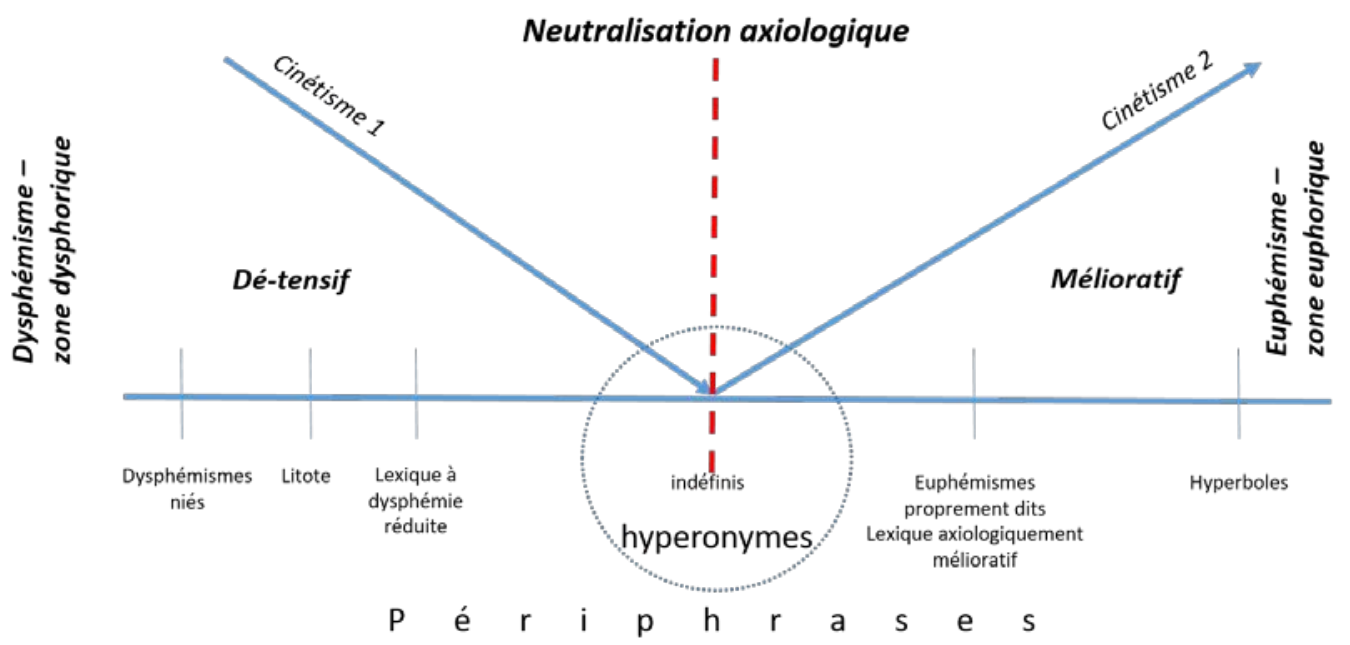

Figure 1 : Diagramme d'euphémisation

Nous attirons l'attention sur le fait que le point de neutralisation axiologique, c'est-à-dire de tension phémique minimale et de mélioration minimale, ne correspond pas à des substantifs " de base ", mais aux expressions indéfinies - généralement dépourvues de contenu axiologique - et aux hyperonymes, nominations généralisantes abondamment utilisées à des fins d'euphémisation, car occultant les traits spécifiques éventuellement dysphémiques dans l'indifférenciation de l'appartenance générique. Dans le cadran du cinétisme détensif décroissant, on peut ranger les dysphémismes niés, les litotes et le lexique à dysphémie réduite, tandis que dans le cadran où le cinétisme mélioratif prend le relais, nous pouvons ranger les euphémismes proprement dits: catachrèses et lexique axiologiquement mélioratif. Le cinétisme mélioratif, non borné, peut arriver jusqu'à l'hyperbole, qui apparaît comme le point ultime de l'euphémisation ${ }^{8}$. Les désignations périphrastiques peuvent bien sûr intervenir à tous les points de ce continuum.

\section{Analyse d'exemples}

Une fois les balises théoriques posées et après avoir défini les outils de l'analyse, nous pouvons maintenant nous consacrer à l'étude multimodale de quelques exemples concrets (Gérard Larcher, $\$ 4$ 4. ; Christophe Castaner, $\$ 4.2$. ), afin de vérifier nos hypothèses et de montrer de quelle manière le conflit nominatif peut être mis au service de l'euphémisation.

\footnotetext{
${ }^{8}$ L'hyperbole constitue même une forme d'inversion de l'euphémisme, car l'atténuation d'un sème ou d'une connotation négative cède le pas à l'amplification d'une caractéristique positive : si le dysphémisme fou peut être euphémisé par original, le superlatif génial peut constituer l'aboutissement de la ligne de mélioration sur laquelle se trouve original aussi.
} 


\subsection{Gérard Larcher}

Le premier extrait que nous proposons est tiré de l'interview, lors de la campagne présidentielle 2017, de Gérard Larcher, du parti Les Républicains, alors président du Sénat et soutien du candidat François Fillon. C'est en effet au sujet de celuici et des affaires judiciaires dans lesquelles il est impliqué que Gérard Larcher est interrogé. Dans cette situation tendue, qui porte atteinte au camp du politique, un journaliste (J4) évoque des faits (les costumes à 6500 euros) en y rattachant la dénonciation d'Alain Chrétien et les réactions des salariés de Peugeot. Cette dénonciation est signalée par l'emploi, a priori neutre, du verbe dire. C'est néanmoins le caractère trop " objectif» et direct de la modalisation énonciative portée par ce verbe qui déclenche le conflit nominatif. Larcher répond en recatégorisant euphémiquement la dénonciation implicite dans le verbe dire par le syntagme une interrogation [...] qui est pas illégitime, dans une structure dont le verbe passe du moyen (se pose) au passif (est posée), ce qui en atténue l'intentionnalité éventuellement malveillante, bien que l'agent n'en soit pas effacé (des ouvriers de chez Peugeot). Ce début de conflit nominatif est relancé par les journalistes, qui contestent le terme interrogation (ça fait quand même beaucoup d'interrogations légitimes), et, bien que J2 s'en excuse ouvertement (pardonnez-moi), il ne renonce pas à sa proposition nominative: les interrogations redeviennent des affirmations et le verbe poser la question redevient dire. Contraint de poursuivre le conflit, le politique reprend alors la parole : après une repartie atténuant les affirmations (objectives) en jugement (subjectif), Larcher produit quelques entassements paradigmatiques (hésitations, amorces de phrases, allongements syllabiques et pauses vides) avant d'aboutir à une nomination euphémique qu'il juge d'autant plus satisfaisante qu'elle constitue une paronomase de la forme rejetée (ils sont plutôt interpellés qu ìnterrogés).

[2]

J4 quand par exemple Alain Chrétien maire euh républicain de Vesoul DIT dans Le Monde d'hier dans ma ville il y a une usine Peugeot je me vois mal dé- je me vois mal défendre les costumes à 6500 euros + vous lui dites quoi à Alain Chrétien

$\mathrm{P}$ que c'est UNE INTERROGATION de la part pas simplement + euh des ouvriers de chez Peugeot qui se pose et qui est posée et QUI EST PAS ILLÉGITIME

J2 mais vous dites ça fait quand même beaucoup d'interrogations légitimes sur votre candidat hein c'est la quatrième depuis le début de: euh ++ de l'interview $X X$

$\mathrm{J} 4$ toujours ++++ oui + vous dites ce sont des interrogations

$\mathrm{P}$ oui mais parce que je suis un homme libre et je soutiens librement François Fillon je pense que c'est l'intérêt de notre pays c'est le seul qui porte

J2 Monsieur Larcher 
$P$ des réformes mais en même temps je n'ai jamais jamais été quelqu'un d'aligné

J2 Monsieur Larcher vous dites ++ et Gilles le rappelait à l'instant 'interrogations' CE NE SONT PAS DES INTERROGATIONS PARDONNEZ-MOI + CE SONT À CHAQUE FOIS DES AFFIRMATIONS + les gens NE POSENT PAS LA QUESTION ils DISENT c'est pas possible +++

P c'est leur JUGEMENT ++ JE NE DIS + PAS QUE: + ÇA NE SOIT PAS S:ANS INTERPELLER + EN FAIT ILS SONT plutôt INTERPELLÉS QU'INTERROGÉS (France Info, 28/03/2017).

Le conflit nominatif implique les verbes dire, poser la question, interpeller, interroger, l'adjectif légitime et les noms interrogation, affirmations, jugement. La figure 2 montre comment se distribuent ces unités sur notre diagramme : la couleur rouge indique les dysphémismes et les mouvements de dysphémie, tandis que le vert indique les euphémismes ainsi que les mouvements d'euphémisation. On remarquera que l'euphémisation se limite ici à réduire la tension : tous les éléments du conflit nominatif se distribuent en effet dans le seul cadran dé-tensif, alors que le cinétisme mélioratif n'intervient pas dans ce cas. Par ailleurs, il y a une répartition stricte entre les locuteurs pour ce qui est de la production des euphémismes et des dysphémismes, ceux-ci étant l'apanage des journalistes, alors que le politique a celui des euphémismes.

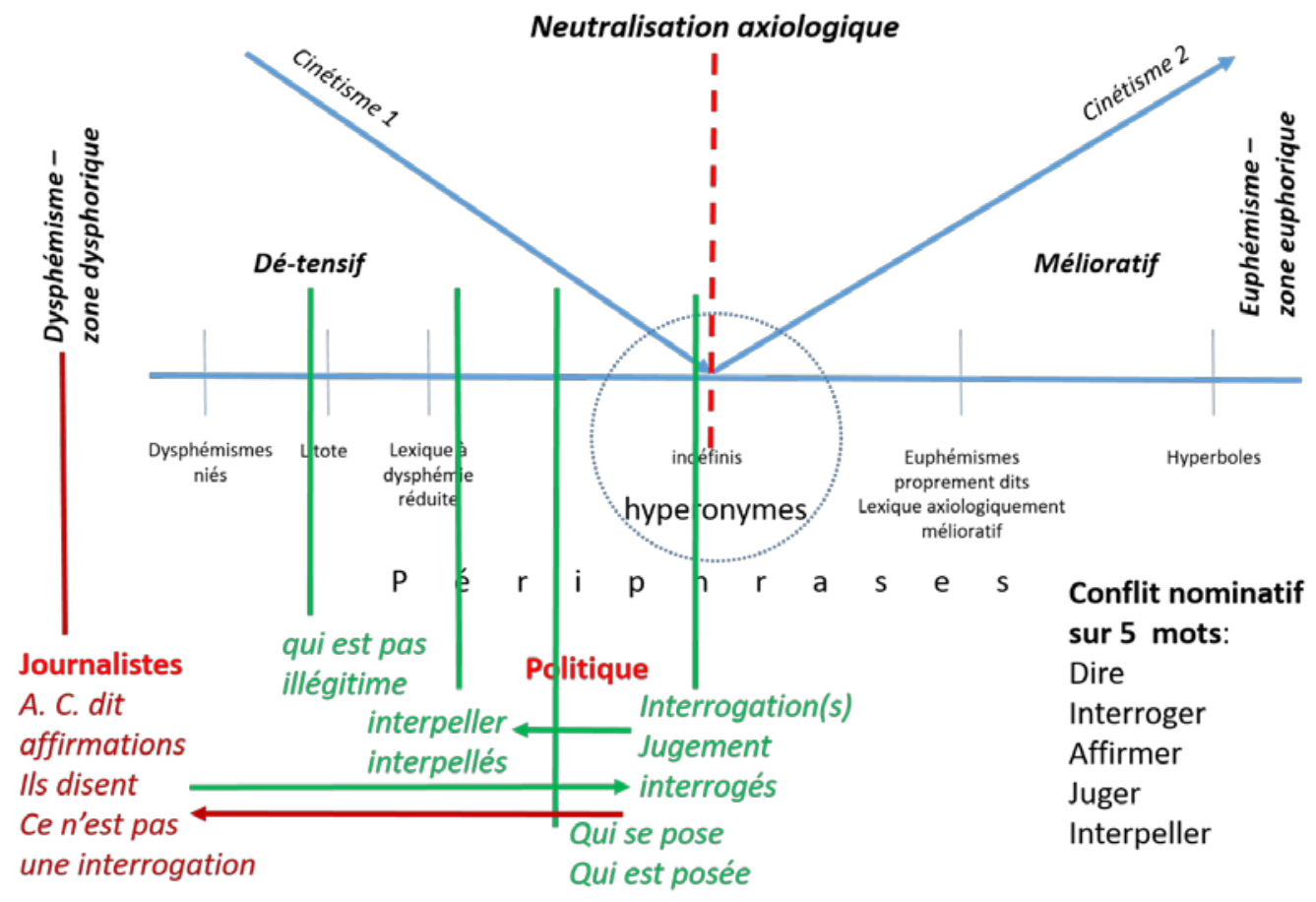

Figure 2 : Conflit nominatif et euphémisation de G. Larcher 
Concernant l'imbrication des plans segmental, supraségmental et coverbal, nous nous focaliserons ici sur la dernière réplique de Larcher car c'est celle qui, d'une part, montre le plus de disfluences et qui, d'autre part, clôt le conflit nominatif par une validation forte du mot interpellés de la part du politique, que celui-ci estime pouvoir être consensuel. En effet, après cette réplique, le journaliste passe à autre chose.

Sur le plan segmental, on remarque la triple négation (je ne dis pas que ça ne soit pas sans interpeller) opacifiant l'opération de référenciation et visant à occuper le temps de parole pendant la recherche (aboutie) de la nomination euphémique susceptible de prendre la place d'interroger / interrogation dans le dialogisme interlocutif : il lui faut en effet 5,3 secondes avant la production d'interpeller, qui est introduit par le marqueur de reformulation plutôt.

Sur le plan suprasegmental, on remarque des stratégies de prise de temps complémentaires de ce que nous avons remarqué au plan segmental : une occlusion totale du canal vocal avant le premier pas (fermeture des lèvres pour la réalisation de la bilabiale /p/, correspondant au blanc dans le spectrogramme), prononcé avec une grande force expiratoire à l'issue d'une pause d'hésitation, le vibrato sur que (forme d'allongement), l'allongement de la sibilante initiale de sans et plusieurs pauses silencieuses. Nous signalons enfin le coup de glotte initial lors de la reprise du verbe interpellés, destiné, dans ce cas, non pas à prendre du temps en vue de la recherche lexicale mais à exercer l'autre fonction capitale de l'intonation : celle d'instaurer une prosodie contrastive avec interrogés.

Sur le plan mimogestuel, enfin, on remarque des gestes de ponctuation rythmique à faible amplitude (main gauche et tête) avant la production de l'euphémisme interpeller, ainsi que la fermeture des yeux, qui constitue un indice d'autocentrage du locuteur qui se coupe un instant de l'interaction afin de se concentrer sur la recherche lexicale. Une fois la nomination trouvée, les yeux rétablissent le contact visuel avec l'interlocuteur, alors que les autres gestes restent de même nature mais que leur amplitude est plus grande. La séquence se termine par un sourire de satisfaction pour la trouvaille euphémique, qui ponctue la conclusion du conflit nominatif. 


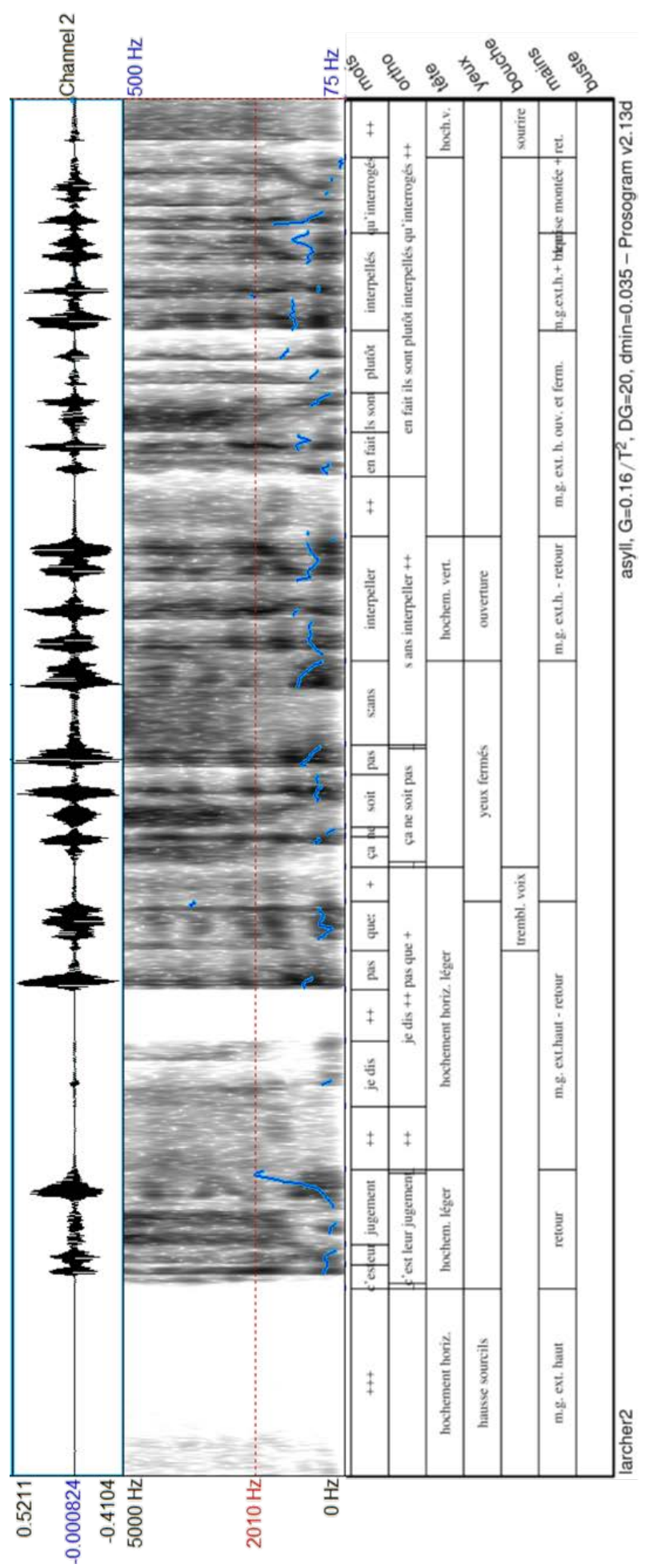

Figure 3 : Tracé prosodique et gestuel de G. Larcher 


\subsection{Christophe Castaner}

Le deuxième extrait remonte à juillet 2017, quelques mois après l'élection d'Emmanuel Macron et le renouvellement de l'Assemblée Nationale. Christophe Castaner est à l'époque porte-parole du gouvernement d'Edouard Philippe et secrétaire d'État chargé des Relations avec le Parlement ; la journaliste l'interroge au sujet de l'actualité politique. Le conflit nominatif est déclenché par l'emploi, de la part du politique, de la métaphore bisounours au détour d'une réponse. Dans cette première occurrence, la métaphore est mise au service d'une litote euphémique : le monde de l'économie n'est pas le monde des bisounours s'appuie sur le sème, axiologiquement positif, de /gentil/ attaché aux personnages des Bisounours (le monde de l'économie est brutal). Or, la journaliste se saisit de cette métaphore, qu'elle va utiliser de façon dysphémique pour caractériser les élus LREM, ce qui suscite bien entendu la réaction de C. Castaner. Dans ce cas, la métaphore est employée de façon ironique, ce qui est signalé à la fois par la question rhétorique et la structure litotique (ils ne sont pas un peu bisounours?) et le sème sélectionné n'est par conséquent plus /gentil/ mais bien /naïf/. Le changement d'orientation axiologique est manifesté, d'une part, par la répétition de bisounours et la caractérisation des députés LREM comme marcheurs (ce n'est plus la République qui est en marche, mais ses partisans qui entrent dans une famille lexicale - celle des marcheurs - pas forcément positive, puisqu'elle contient virtuellement des nominations telles que promeneurs, flâneurs, glandeurs...) ; d'autre part, par une autre métaphore axiologiquement négative complétée par une litote : ça flotte un peu - un peu beaucoup même. Castaner ne poursuit pas immédiatement le conflit nominatif: d'abord il donne une réponse qui argumente en faveur de l'efficacité de ces députés et ce n'est qu'ensuite qu'il oppose une nomination euphémique pour catégoriser les épisodes auxquels la journaliste fait allusion lorsqu'elle emploie l'expression litotique ça flotte un peu. Comme on peut le voir aussi dans notre diagramme, le politique utilise d'abord le mot à dysphémie réduite maladresses, puis des désignations indéfinies et indirectes (moments qui vont passer en boucle à la télé) et des métaphores allusives (se prend les pieds dans le tapis) dont il cite la source énonciative, qui s'assimile au ON-discours doxal (comme on dit: marqueur de reformulation), comme une sorte de caution, de validation de son langage. Ce n'est qu'après ces touches qui complètent le cinétisme dé-tensif que le politique s'autorise un terme mélioratif, l'euphémisme femmes et hommes nouveaux (pour "inexpérimentés »).

J partenaires italiens qui ont pas vraiment apprécié

P (expiration) c'est le principe de la négociation vous savez le monde de l'économie c'est un peu comme le monde de la politique c'est pas le monde des BISOUNOURS + de temps en temps faut l'assumer 
J alors puisqu'on parle des: des BISOUNOURS justement euh est-ce que vous qui êtes en charge des relations avec le parlement euh vos députés marcheurs ils ne sont pas un peu BISOUNOURS o:n a le sentiment quand on les voit à l'assemblée nationale que ++++ ÇA FLOTTE UN PEU

$\mathrm{P}$ vous savez moi + comme vous l'avez dit comme

J UN PEU BEAUCOUP MÊME

$\mathrm{P}$ je suis ministre en charge des relations je regarde le calendrier

J oui

$\mathrm{P}$ on avait envisagé euh que la session extraordinaire pour voter tous les textes qu'on y a inscrits se termine le dix j'avais même pensé un moment le dix-huit août + je pense qu'elle se terminera la semaine prochaine + donc au bout du compte ce qui compte c'est l'efficacité alors oui: il y a des MALADRESSES + oui il y a des

$\mathrm{J}$ alors ça

P MOMENTS EUH QUI VONT: PASSER EN BOUCLE À LA TÉLÉ et $j$ 'ai vu le reportage juste avant que vous me receviez avec une vice-présidente qui À UN MOMENT DONNÉ EUH SE PREND COMME ON DIT LES PIEDS DANS LE TAPIS mais + vous savez c'est aussi le risque de demander à DES FEMMES ET À DES HOMMES NOUVEAUX de prendre des responsabilités (BFM et RMC, 12/07/2017).

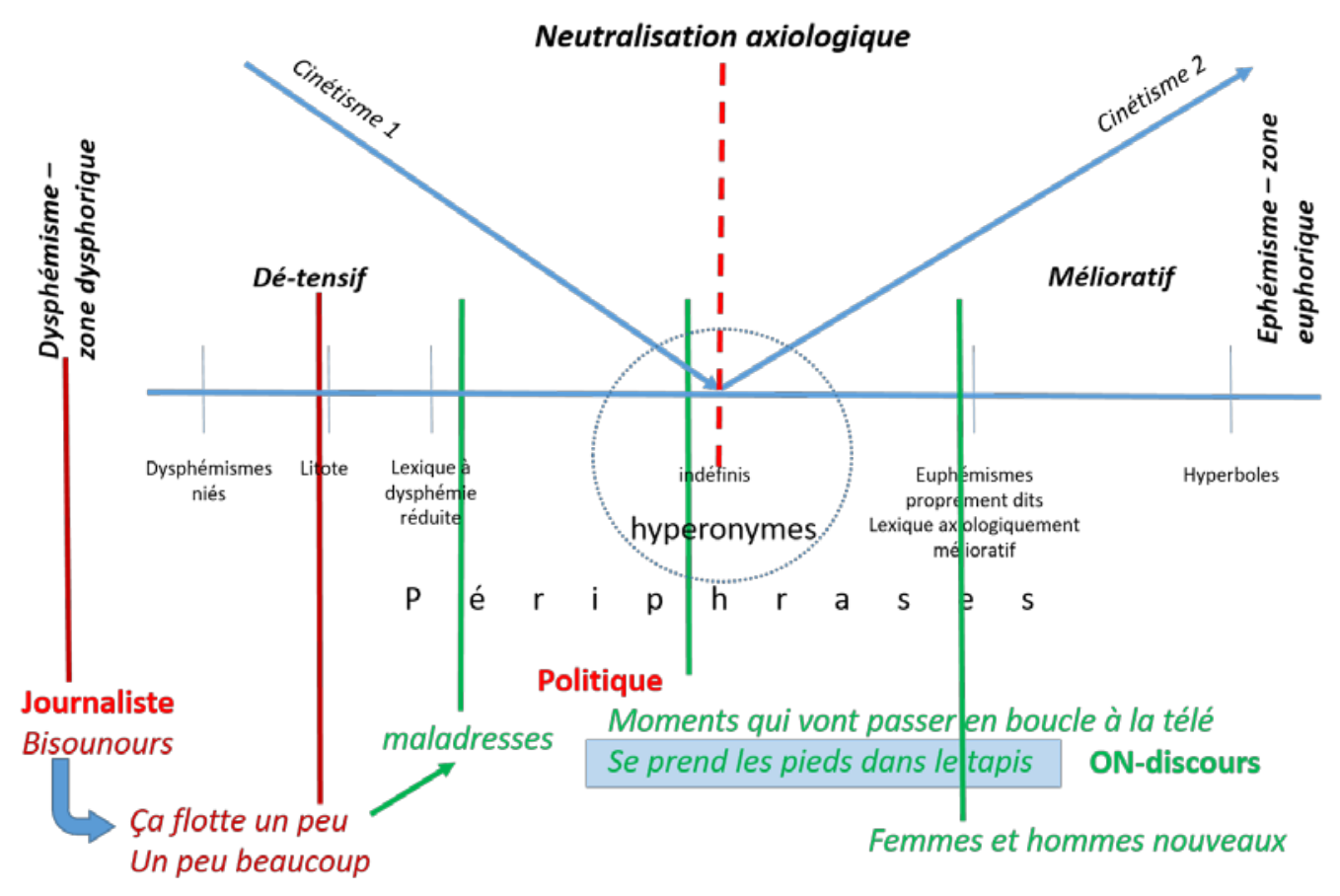

Figure 4 : Conflit nominatif et euphémisation de C. Castaner 
Venons-en maintenant à la manière dont s'articulent les plans segmental, suprasegmental et coverbal dans cette partie finale du conflit nominatif : du point de vue segmental, cette séquence où se déploie l'euphémisation est de type concessif (alors oui il y a des maladresses oui il y a des moments... mais vous savez...) et l'on voit bien que la nomination est basée sur un dialogisme interdiscursif : Castaner essaie de parler avec les mots de l'autre ou, plus précisément, d'une doxa dans laquelle est censée être comprise la journaliste aussi, mais il sélectionne dans cette doxa la nomination la moins dysphémique, donc la moins nuisible pour son camp. La recherche lexicale est témoignée par la récursivité paradigmatisante de la structure il y a ... qui aligne des candidats lexicaux de plus en plus adéquats à obtenir un consensus (entassement paradigmatique). Du point de vue suprasegmental ${ }^{9}$, on remarque que la séquence concessive comporte une amplitude nettement plus large (en termes d'intensité) par rapport au contexte gauche, tandis que la fréquence fondamentale (F0, la courbe prosodique) est globalement moins élevée, ce qui s'accorde avec la phase de recherche lexicale. Du point de vue coverbal, enfin, on constate deux types de geste : d'une part, des cillements fréquents pouvant être rattachés soit à un autocentrage du locuteur qui effectue la recherche lexicale, en vue de la programmation de son dire, soit à une forme de malaise pour la concession qu'il est obligé de faire. D'autre part, on a une augmentation du nombre de segments corporels en mouvement, en particulier les mains, qui ponctuent le discours et dessinent des cercles. Ces gestes ont une fonction phatique, de recherche de connivence avec son interlocuteur au moment où la concession fait entrer le discours de l'autre dans son propre discours. Il s'agit en effet d'un moment extrêmement dangereux, car la concession peut mettre en valeur le locuteur, qui se présente comme un individu tolérant et faisant place aux arguments de l'autre, ou être considérée comme un aveu vis-à-vis des insinuations de la journaliste. De ce point de vue, on remarquera que le geste iconique d'arrondissement des lèvres au moment de la séquence concessive (la «bouche en cœur ») est particulièrement appuyé, dans le but non seulement d'attirer l'attention et la connivence de l'interlocuteur (en effet, ce geste permet de modifier l'émission du son, dont la fonction d'appel se trouve renforcée), mais aussi d'atténuer mimiquement l'importance de la concession formulée verbalement, par une moue exprimant conventionnellement une certaine forme de condescendance.

\footnotetext{
${ }^{9}$ Dans ce tracé prosodique, nous n'avons pas inséré le spectrogramme pour des raisons de lisibilité et parce que dans cet énoncé il n'y a pas de phénomène significatif à ce niveau.
} 


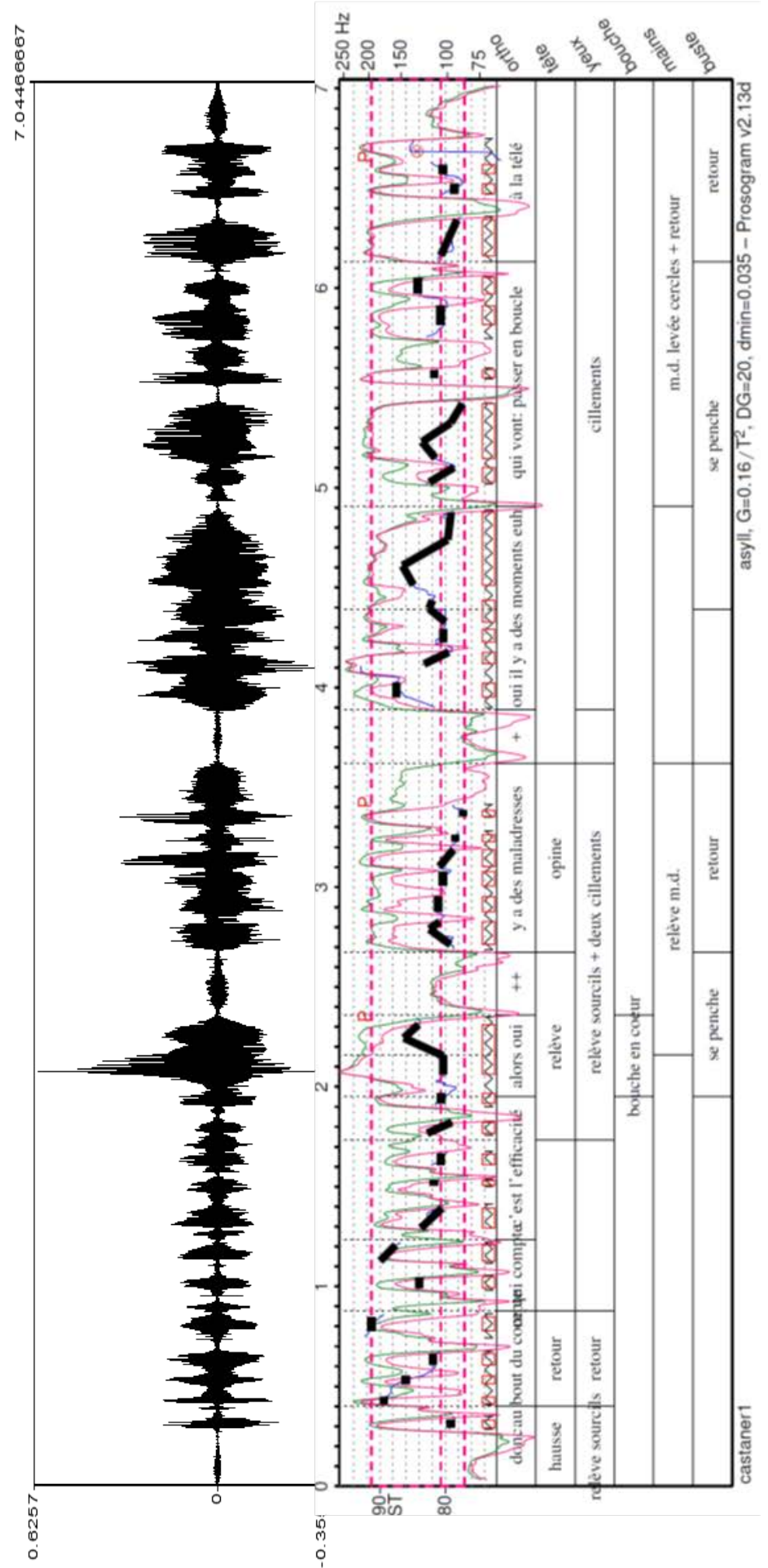

Figure 5 : Tracé prosodique et gestuel de C. Castaner 


\section{Conclusion}

À travers l'analyse de quelques exemples d'euphémisation, nous avons montré que le conflit nominatif est un site interactionnel privilégié d'observation de la polyphonie énonciative : en effet, celui-ci concentre et révèle, en les faisant contraster, les différents PDV en jeu, disponibles et actifs à un moment donné et par rapport à un sujet et à des énonciateurs donnés. Dans l'interview politique, cela constitue même une stratégie de questionnement que le journaliste utilise à bon escient pour mettre à l'épreuve le politique : celui-ci peut en effet réagir de façon colérique ou s'engager dans la négociation d'une nomination euphémique consensuelle, à travers une argumentation qui peut l'amener à faire des révélations malgré lui. Dans les exemples que nous avons analysés, c'est toujours la négociation qui se met en place : ses enjeux éthotiques sont en effet très importants pour le politique, qui ne doit pas s'emporter (eunoia), ni se dérober (aretê), mais au contraire faire preuve de sérénité et de bon sens (phronésis). Lorsque le politique parvient à "gagner" le conflit, à imposer sa propre nomination, cela met en valeur sa personne, le PDV dont il est porteur, ainsi que ses qualités de négociateur.

Par ailleurs, le suprasegmental et le coverbal apparaissent comme des compléments essentiels du conflit nominatif, à valeur diagnostique et fonctionnelle, dans la mesure où ils constituent, pour l'orateur, des moyens de saillance additionnelle de la figuralité (Actio oratoire : dimension fonctionnelle), alors que leur valeur diagnostique permet au chercheur de se baser sur ces éléments en tant que symptômes d'un travail dialogique lors de la recherche lexicale et de l'évaluation des PDV respectifs (perturbation du phrasé, lenteur du débit, déconnexion du contact visuel avec l'interlocuteur), alors que d'autres symptômes (coup de glotte, retour à un débit normal, sourire, rétablissement du contact visuel, etc.) indiquent l'issue positive du conflit. Le dialogisme apparaît ainsi comme une interaction dynamique et évolutive de points de vue au sein de l'interaction orale, et pas simplement comme un rapport de places statiques.

\section{RÉFÉRENCES BIBLIOGRAPHIQUES}

AUCHLIN, Antoine (2000) : "Ethos et expérience du discours : quelques remarques ", in M. Wauthion \& A. C. Simon (éd.), Politesse et idéologie. Rencontres de pragmatique et de rhétorique conversationnelles. Louvain, Peeters, 77-95.

Bonhomme, Marc (2005) : Pragmatique des figures du discours. Paris, Champion.

BOUVET, Danielle \& Mary-Annick MOREL (2002) : Le ballet et la musique de la parole. Le geste et l'intonation dans le dialogue oral en français. Paris, Ophrys.

ClARK, Eve Vivienne (1997) : "Conceptual perspective and lexical choice in acquisition". Cognition, 64, 1-37.

DÉTRIE, Catherine (2001) : Du sens dans le processus métaphorique. Paris, Honoré Champion. 
DRUETTA, Ruggero (2009) : "Métalangage et prosodie de l'atténuation à l'oral ». Synergies Italie, $\mathrm{n}^{\circ}$ spécial [R. Druetta \& P. Paissa, dir., Euphémismes et stratégies d'atténuation du dire], 95-110.

DRUETTA, Ruggero (2015) : "L'hyperbole performée : remarques à partir d'un corpus d'entretiens politiques ». TRANEL, 61-62, 129-151.

DUCROT, Oswald (1984) : Le Dire et le dit. Paris, Minuit.

FREGE, Friedrich Ludwig Gottlob (1892) : «Über Sinn und Bedeutung». Zeitschrift für Philosophie und philosophische Kritik, 100, 22-50.

GERDES, Kim \& Sylvain KAHANE (2009): "Speaking in piles: Paradigmatic annotation of French spoken corpus ». Processing of the fifth Corpus Linguistics Conference, Liverpool, $1-16$.

GUAÏTELLA, Isabelle (1995) : "Mélodie du geste, mimique vocale?». Semiotica, 103 (3/4), 253-76.

Guillaume, Gustave (1929) : Temps et verbe. Théorie des aspects, des modes et des temps suivi de L'architectonique du temps dans les langues classiques. Paris, Champion.

Guillaume, Gustave (1973) : Langage et science du langage. Paris-Québec, Nizet-Laval.

JAUBERT, Anna (2008): "Dire, et plus ou moins dire. Analyse pragmatique de l'euphémisme et de la litote ». Langue française, 160, 105-116.

KLEIBER, Georges (1984) : "Dénomination et relations dénominatives ». Langages, 76, 77 94.

KRIPKE, Saul (1972) : "Naming and Necessity », in Donald Davidson \& Gilbert Harman, (ed.), Semantics of Natural Language. Dordrecht, Reidel, 253-355.

LÓPEZ DÍAZ, Montserrat (2014) : "L'euphémisme, la langue de bois et le politiquement correct : changements linguistiques et stratégies énonciatives ». L'Information grammaticale, 143, 47-55.

MARTIN, Philippe (2011): "Souveraineté-Association en linguistique: l'exemple de l'intonation et de la (macro) syntaxe ", in R. Druetta (éd.), Claire Blanche-Benveniste. La linguistique à l'école de l'oral. Sylvain-les-Moulins, Gerflint, 57-64.

RABATEL, Alain (2003) : "Le point de vue, entre langue et discours, description et interprétation : état de l'art et perspectives ». Cahiers de Praxématique, 41, 7-24.

RABATEL, Alain (2005) : "Le point de vue, une catégorie transversale ". Le français aujourd'hui, 151, 57-68.

Russell, Bertrand (1905) : "On Denoting ». Mind, New Series, 14 (56), 479-493.

SHRIBERG, Elizabeth E. (1994) : Preliminaries to a Theory of Speech Disfluencies. PhD thesis. Berkeley, University of California.

SiBlOT, Paul (2001) : «De la dénomination à la nomination ». Cahiers de praxématique, 36, 189-214.

STRawson, Peter Frederick (1950) : "On Referring ". Mind, New Series, 59 (235), 320344.

TURCHET, Philippe (2009) : Le langage universel du corps. Comprendre l'être humain à travers la gestuelle. Montréal, Éditions de l'Homme. 